\title{
Current Status and Prospects of Reliability Test Research on Special Computers
}

\author{
Chao $\mathrm{Li}^{1, *}$ \\ ${ }^{1}$ Department of Computer, Jiangsu Automation Research Institute, Lianyungang, China
}

\begin{abstract}
Special computers are widely used in aerospace, ships, weapons and other fields. More and more attention is paid to the reliability of special computers. Although my country started late in this regard, it has achieved some results after continuous exploration by a large number of scientific research institutions, and has gradually kept up with the pace of research in developed countries. This article first summarizes the research status and importance of accelerated life test in reliability test research, and analyzes the key technology and development of reliability test of special computer. Reliability analysis can solve the failure problems that cannot be explained by traditional quality analysis methods, and will surely make remarkable achievements in the future industrial development. The summary of this article will provide theoretical reference for subsequent related research.
\end{abstract}

\section{Introduction}

The reliability of electronic products is one of the key factors affecting the quality of their products, and with the large-scale control system, involving many electronic components, the failure of a single component will affect the normal operation of the entire system. Therefore, in recent years, more and more attention has been paid to the reliability of components, and a large number of scientific research institutions have carried out related research [16]. It is necessary to test the reliability of electronic products in a predetermined environment and meet the reliability indicators. The developed countries in the world have closely integrated reliability technology and total quality management to improve the reliability of products.

Levenbach published "Accelerated Life Test of Capacitors" in 1957. The project of accelerated life test was established with its as a sign and began to be established as an extremely applicable subject. Compared with life test under normal environment, accelerated life The advantages of the test: the test time is very short, the test conditions and results are easy to control, the test is convenient, etc. Therefore, the accelerated life test technology has been rapidly applied and developed in the engineering, military and aerospace industries at home and abroad. In the military field, some developed countries Using advanced technology, developed a missile weapon storage life extension accelerated depreciation test; in the civilian field, the development and application of accelerated life test played an active role in the early life test of products, improving product quality, optimizing maintenance strategies, etc. Both have made great contributions.

Accelerated life testing in my country only started in the 1970s, and it has gradually developed, especially for applications in missiles and aerospace, machinery, electronics and other industries. Due to the late development of this test in my country, coupled with the backward equipment, there is still a big technological gap between my country's accelerated life test and some foreign developed countries.

The theoretical research of accelerated life test includes two parts: optimization design and statistical analysis. Under the conditions of a given range of accelerated stress levels, how to determine the sample size and the number of products tested under different stress levels to obtain a more optimally designed accelerated life test. Experts at home and abroad study optimization design problems, mainly in the constant accelerating test of exponential distribution, Weibull distribution and lognormal distribution.

The study of statistical methods for accelerated life testing began in the 1960s, while my country only started in the 1970s.

\section{The key technology and development of special computer reliability test}

Long-life analog integrated circuits usually refer to analog integrated circuits with a life span of more than 10 years. This type of circuit has a high level of reliability and can maintain normal functions for a long time. The existing long-life evaluation tests of analog integrated circuits have two methods: normal stress life evaluation and accelerated stress life evaluation. The accelerated stress evaluation includes accelerated life test ALT (Accelerated Life Testing) and accelerated degradation test ADT (Accelerated Degradation Testing). Two methods. The

*e-mail: sansking@163.com 
normal stress life assessment test means that the device is placed in actual environmental conditions and normal working or storage stress is applied; the accelerated stress life assessment is to increase the product storage or working stress conditions to accelerate the failure of the product in a short period of time and obtain acceleration Product life information under stress conditions, and through corresponding acceleration models, extrapolate product storage or working life under normal stress conditions [9-16]. Normal stress life is the most direct method to evaluate the long life of analog integrated circuits. The life data is the most authentic, but the test cycle is long, the cost is high, and it is no longer suitable for the short development cycle of equipment systems. Accelerated stress life assessment can obtain product life information under normal storage or working conditions in a short period of time, and has become an effective long-life assessment method. Although the accelerated life test evaluation method has a long research history, and the processing of related life data is relatively easy. However, according to a large number of research practices that our institute has carried out in recent years in the long-life evaluation of high-reliability analog integrated circuits, it is found that accelerated stress tests on high-reliability long-life analog integrated circuits often result in very few product failures, or even failures. The product has failed. If you want to obtain sufficient failure data, it can only be achieved by increasing the number of samples or the test time, which makes the test cost and cycle increase sharply. The use of life evaluation technology based on parameter degradation can provide more information about reliability and solve the problem of life evaluation caused by non-failure data when the test device does not need or rarely needs to fail during the test. For slow-degrading high-reliability, long-life analog integrated circuits, the performance degradation process can be accelerated by increasing certain stress levels. Compared with the traditional life test, the life evaluation technology based on parameter degradation can provide more accurate life estimation with degradation data, and it is more reliable and reasonable.

\subsection{Statistical analysis of constant plus test}

Hengjia test statistics has been studied a lot, and the methods have been basically mastered. China has issued a series of national standards such as GJB92-86, GJB73691, GJB2689-81, etc. Constant addition test statistical analysis is to directly use test data, so the accuracy of component statistical analysis can be improved.

\subsection{Statistical analysis of step plus test}

The step-by-step test is mainly to obtain the failure data from the product under a certain stress level, and then convert it to the failure time under another stress level. In 1980, Nelson proposed a well-known cumulative damage model. Under different stress levels in the same test, the failure time of the test can be converted to each other through the failure distribution function. This breakthrough makes the step plus test very researchable.
Domestic scholars such as Wang Lingling, Zhu Shisong, etc. have studied the statistical analysis methods of stepby-step tests for lognormal distribution, Weibull distribution and exponential distribution in the "Accelerated Life Test".

\subsection{Statistical analysis of sequential addition test}

Due to the complexity of the test equipment, the sequential addition test is rarely used in engineering. my country has made great achievements in the sequential addition test. Fei Heliang gives statistics on the accelerated test methods for Weibull distribution and lognormal distribution. The analysis has been widely used, such as in solid-state electrolytic capacitors.

Russia's accelerated storage test has been widely used in the field of missile weapon development and has achieved impressive results. For example, the Torch Design Bureau has established a set of accelerated tests, including test principles, equipment, software and methods, which can not only test components and raw materials, but also test equipment and systems. Russian experts call it their own way to calculate test methods. "Calculation" refers to statistical analysis, and "testing" refers to engineering experiments.

From "Statistical Analysis of Step Stress Accelerated Life Test under Exponential Distribution", the method of reliability and accelerated life test was introduced systematically. Zhang Jianping made a statistical analysis on the use of accelerated life test of liquid crystal display. In 1985, Wan Shisong et al. The application of distribution in constant plus and step plus experiments introduced many methods. In 1995, Zhou Yuanquan and Weng Chaoxi made statistical inferences on factor analysis. In 1992, Ge Guangping and Ma Haixun discussed the parameters of Weibull distribution in step plus experiments. After statistical analysis, Zhou Yuanquan, Zhu Xinwei, etc. put forward a new direction on reliability growth. In 2002, Liu Ruiyuan carried out the most effective design in predecessors' step-increasing test, which was published in "The Optimal Design of StepStress Accelerated Life Test" Inferred the timing (or timing) censoring test of the data in the accelerated life test, thereby making the accelerated test theory more perfect. Sun Zhanquan, Huang Hongzhong, etc. conducted a reliability study on the gears that obey the logarithmic normal distribution, 2005 Geng Xinmin and Zhang Jianping conducted research on the life test of the vacuum fluorescent display. Dai Shusen and Fei Heliang mainly introduced some reliability statistical analysis; Zhou Yuanquan talked about the product in the accelerated life test, too high stress will cause the product to appear new failure chemistry, so in the test process, should ensure that the stress level is not Too high; "Approximate Confidence Region of Several Distribution Parameters under Timed Censoring Life Test" introduces how to calculate the approximate confidence limits of products subject to different distributions under the timed end test. 


\section{Development status of highly accelerated screening tests}

\subsection{The development process of highly accelerated screening tests}

The reliability of the product can be guaranteed at the beginning of the design, but in the production process, the difference in raw materials, production technology, processing equipment, and the technical level of personnel causes more or less defects and hidden dangers in the product. Obvious defects can be passed through routine If the potential defects are not eliminated, the product will often have early failures during use, which will make the reliability of the product lower than that of the conventional product and cannot meet the requirements at the beginning of the design.

In the initial stage of environmental screening, the method generally used is to apply simple vibration shock or temperature, such as drop test, aging test, etc. However, this method has obvious disadvantages, that is, the stress cannot be quantified and the repeatability is poor. In the 1950s and 1960s, the United States proposed the AGREE method (combination of temperature cycling and fixed string vibration), which is much better than simply applying vibration or temperature. However, with the overall improvement of the industrial level, especially the level of components, the environmental resistance of products is getting stronger and stronger, and this method is obviously not enough. In the 1970s, the US Navy proposed environmental stress screening (also known as high-efficiency environmental stress screening at the time), which is a comprehensive test of temperature cycling and vibration. And this method was successfully applied to the Apollo moon landing program, and later applied to the Poseidon missile.

Through $100 \%$ environmental stress screening of products, early failures caused by design, device materials, processing and other reasons are eliminated, so as to shorten the cycle of high product failure rate and reduce the failure rate of products to the specified rate as soon as possible Level, so as to ensure the reliability of the product. However, a large number of tests have proved that for some special and complex products, even after the ESS screening, there are still a large number of failures in the actual use of the products, and the expected screening effect is not ideal.

In the 1980s, Dr. Greggk K. Hobbs from the United States proposed the HASS test. The HASS test screens products with a stress value much higher than the normal stress of the product, which can eliminate a large number of potential defects of the product in a relatively short time, and can effectively overcome the low efficiency of conventional tests. Beginning in the 1990s, HASS has been rapidly promoted and applied worldwide, especially in the field of civil electronics, which has grown exponentially.

\subsection{Current research status of highly accelerated screening tests at home and abroad}

The overall quality of the product is evaluated through the HASS test, so that process defects and weaknesses can be detected in the pilot phase, which greatly improves the department's product profitability. John A Hansen applies the HASS test method to the medical device industry to optimize the reliability of the equipment and avoid the catastrophic consequences caused by the failure of key parts of the medical equipment. At the same time, it is used as a tool to meet various risk management requirements of the international community. Neill Doertenbach et al. used the classic fatigue calculation method to calculate the life loss of the product after the HASS test, and used a conservative calculation result to prove that the HASS test will not excessively lose the life of the product. Balaji Janamanchi uses a financial model to weigh the relevant implementation costs and benefits of HASS. HASS not only increases the mean time between failures of the product, but also contributes to the improvement of product design and production process, and saves costs. He believes that HASS can enable manufacturing The business will benefit better.

Around 1950, HASS technology began to attract the attention of domestic scholars, and researches on the principles and related applications of HASS experiments were carried out. Zhu Yaochang explained that the advantage of high-accelerated stress test is that through high-stress screening, the product itself can be guaranteed to have high reliability, and the efficiency of the test can be used to greatly shorten the product development cycle, thereby reducing development costs. By analyzing the application of this technology in various fields (such as machinery, electromechanics, electronics, etc.) at home and abroad, he believes that this technology is worthy of promotion and application. After research and analysis, Deng Lin pointed out that HALT/HASS is very suitable for miniaturized products with simple functions due to its high test efficiency. Because of its simple structure and few failure modes, it is easy to learn from each other's successful experience. Chu Weihua and others analyzed the basic principles of the HALT/HASS test, and summarized the HALT/HASS test method and the process of establishing the test profile.

In order to identify and eliminate early product failures and achieve the best results at the lowest cost, the School of Reliability and System Engineering of Beihang University, taking circuit board solder joints as an example, proposed a HASS test method based on computer simulation technology. Peng Li and others first proposed applying the simulation method to the HASS test, and conducted a simulation-based HASS test design for a certain type of circuit board, which has a certain degree of practicability. Since HALT/HASS has significantly improved the quality and reliability of products, and has greatly saved costs, most companies restrict the disclosure of related application results, plus foreign confidentiality of this advanced technology, and publicly published related documents on HASS technology There are very few, so the domestic HASS technology is in the initial application state of tracking and typical products. Related 
research and application still require continuous efforts in reliability work.

\section{Conclusion}

After more than half a century of development, reliability analysis methods have been fully understood and used in the electronics, aerospace and aviation industries, and reliability research has played a very important role in both the economic and safety fields. First of all, the theory and mathematical models of reliability analysis have gradually matured. Commonly used mathematical models include exponential distribution, normal distribution, Weibull distribution, etc. These distribution functions can effectively describe the reliability of products with their different mathematical characteristics. Secondly, the reliability analysis method has developed from simple failure mathematics statistics to the study of failure mechanism. For example, the most commonly used methods such as stress-strength interference theory and cumulative damage theory in engineering, these methods can quantitatively analyze the failure process of the product by studying the physical properties of the product. It also formed a systematic failure data processing method, that is, the reliability analysis of the failure system. Commonly used methods include the first developed failure mode, impact and criticality analysis (FMECA) and fault tree analysis (FTA). And probabilistic risk assessment (PRA) developed on this basis. These methods are based on the structural characteristics of the product system itself, using various logical reasoning methods, combined with mathematical operations, to realize the overall reliability evaluation of the system and make the reliability analysis more comprehensive. Reliability is a quantitative description of the normal working ability of a product, and an index to measure the quality of the product. Reliability analysis can solve the failure problems that cannot be explained by traditional quality analysis methods, and will surely make remarkable achievements in the future industrial development.

\section{References}

1. Li Jing. Research on Accelerated Life Test Method and Application of Electronic Equipment[J]. Electronic Quality. 2020(3): 24-28.

2. Li Zhe, Meng Li, Xu Guangning, et al. Application research of highly accelerated life test on optical fiber acousto-optic modulator[J]. Equipment Environmental Engineering. 2020, 17(3): 78-83.

3. Long Bing, Zhang Zhongzhan. Reliability analysis of constant stress accelerated life test $[\mathrm{J}]$. Firepower and Command Control. 2020, 45(1): 53-58, 64.

4. Ni Jing, Shi Yu, He Lihua. Design of Accelerated Experiment System for Reliability of Robot Electrical Connectors[J]. Experimental Technology and Management. 2020, 37(2): 95-99.

5. Chen Wenhua, He Qingchuan, Pan Jun, et al. Research status and prospect of reliability test technology of mechanical products[J]. China
Mechanical Engineering. 2020, 31(1): 72-82.

6. Wang Li, Gu Xiaohui, Pan Shouhua. Two-parameter accelerated degradation law analysis of an O-ring seal[J]. Equipment Environmental Engineering. 2019, 16(11): 84-89. 